\title{
Proving the Goldbach's conjecture
}

\author{
Ninh Khac Son \\ Date Performed: 06 December 2019
}

\begin{abstract}
Goldbach's conjecture is one of the oldest and best-known unsolved problems in number theory and all of mathematics. It states:
\end{abstract}

"Every even integer greater than 2 can be expressed as the sum of two primes". Manuscript content: Prove that Goldbach's conjecture is correct.

Key words: Prime numbers, Goldbach's conjecture, number theory.

\section{Notation system}

We briefly mention the symbols and theorems in number theory to apply to this manuscript.

\subsection{Notation}

- Symbol of positive natural number: $N^{*}$

- Symbol of prime number greater than 2: $\mathbf{P}^{*}$

- Symbol of odd-number greater than 2: $\mathrm{O}^{*}$

\subsection{The operations express odd and prime numbers}

- For every odd natural number $\mathbf{O}$ greater than 2 , it can always be expressed as:

$$
O=2 n+1\left(\text { With }: O \in O^{*}, n \in N^{*}\right)
$$

This deduces the result: For every odd natural number $\mathbf{O}^{\prime}$ greater than 5 , it can always be expressed as:

$$
O^{\prime}=2 n^{\prime}+P\left(\text { With }: n^{\prime} \in N^{*}, P \in P^{*}, P<O^{\prime}\right)
$$


This also deduces the result: For every prime number $\mathrm{P}$ greater than 5, it can always be expressed as:

$$
P=2 m+P^{\prime}\left(\text { With }: m \in N^{*}, P^{\prime} \in P^{*}, P^{\prime}<P\right)
$$

\subsection{Bertrand's postulate}

Bertrand's postulate is a theorem stating that for any integer $n>3$, there always exists at least one prime number $\mathrm{p}$ with

$$
n<p<2 n-2
$$

\section{Goldbach's conjecture}

Goldbach's conjecture is one of the oldest and best-known unsolved problems in number theory and all of mathematics. It states:

"Every even integer greater than 2 can be expressed as the sum of two primes".

\section{Proving the Goldbach's conjecture}

3.1. Consider even integer numbers $2<N \leq 10$

- For $N=4$, represent: $N=2+2$

- For $N=6$, represent: $N=3+3$

- For $N=8$, represent: $N=3+5$

- For $N=10$, represent: $N=3+7$

\subsection{Consider even integer numbers $N>10$}

Because $\mathrm{N}$ is an even integer greater than 10, $\mathrm{N}$ can always be expressed as the sum of two odd numbers:

$$
N=O_{1}+O_{2}\left(\text { With : } O_{1}, O_{2} \in O^{*}, O_{1}<O_{2}\right)
$$

Because the prime number $P_{1}$ is greater than 2 in the set $P^{*}$, it also belongs to the set $O^{*}$. Therefore, $\mathrm{N}$ can always be expressed as the sum of a prime number $P_{1}$ and an odd number $O$ :

$$
N=P_{x}+O\left(\text { With }: \forall P_{x} \in P^{*}, O \in O^{*}, P_{x}<N / 2\right)
$$


Based on the result of (2), we can express the odd number $\mathrm{O}$ to:

$$
\left.O=P_{x}+2 n \text { (With }: n \in N^{*}, P_{x} \in P^{*}, P_{x}<O\right)
$$

Finally, this is stated as follows: With every even natural number $N>10$, there is always at least a prime number $P_{1}$ in the set $P^{*}$ such that

$$
\left.N=P_{x}+\left(P_{x}+2 n\right) \text { (With }: P_{x} \in P^{*}, n \in N^{*}, P_{x}, n<N / 2\right)
$$

Example: Any even natural number $N$ greater than 10, it can be expressed by $\forall P_{x}<N / 2$ or $N=P_{x=\text { const }}+\left(P_{x=\text { const }}+2 n\right)$.

From expression (7), we transform to produce the result:

$$
n=\frac{N}{2}-P_{x}\left(\text { With }: P_{x} \in P^{*}, n \in N^{*}, P_{x}<N / 2\right)
$$

In particular, $\mathrm{N}$ has a given value, so the value of $\mathrm{n}$ will vary with $P_{x}$. While $P_{x} \in P^{*}$, we convert $P_{x}$ into the set $\mathrm{N}^{*}$ to construct a function $\mathrm{f}$ :

$$
f=\frac{N}{2}-x\left(\text { With }: x \in N^{*}, x<N / 2\right)
$$

Thus, the value of $\mathrm{f}$ contains the value of $\mathrm{n}$ and the value of $\mathrm{x}$ contains the value of $P_{x}$. This means that the values of $\mathrm{n}$ and $P_{x}$ always belong the graph of the function $\mathrm{f}$.

On the other hand, based on the expressions (5) (6), since $N / 2<P_{x}+2 n<$ $N$, this results in the value of $\mathrm{n}$ also in the graph of the function $\mathrm{g}$ :

$$
g=x-\frac{N}{2}\left(\text { With }: \quad x \in N^{*}, N / 2<x<N\right)
$$

Thus, the value of $\mathrm{g}$ contains the value of $\mathrm{n}$ and the value of $\mathrm{x}$ contains the value of $2 n+P_{x}$. This means that the values of $\mathrm{n}$ and $2 n+P_{x}$ always belong the graph of the function $\mathrm{g}$.

Purpose of functions: We construct the functions $f$ and $g$ to apply the Bertrand's theorem to the $x$ value of those two functions to find the prime numbers in the set $(N / 2, N-1)$. In which, the $x$ value of the function $f$ belongs to the set $x \in(0, N / 2)$ and the $x$ value of the function $g$ belongs to the set 
$x \in(N / 2, N-1)$ when these two functions are on the same coordinate system.

Graphing two functions $\mathrm{f}$ and $\mathrm{g}$ on the same coordinate system, we have:

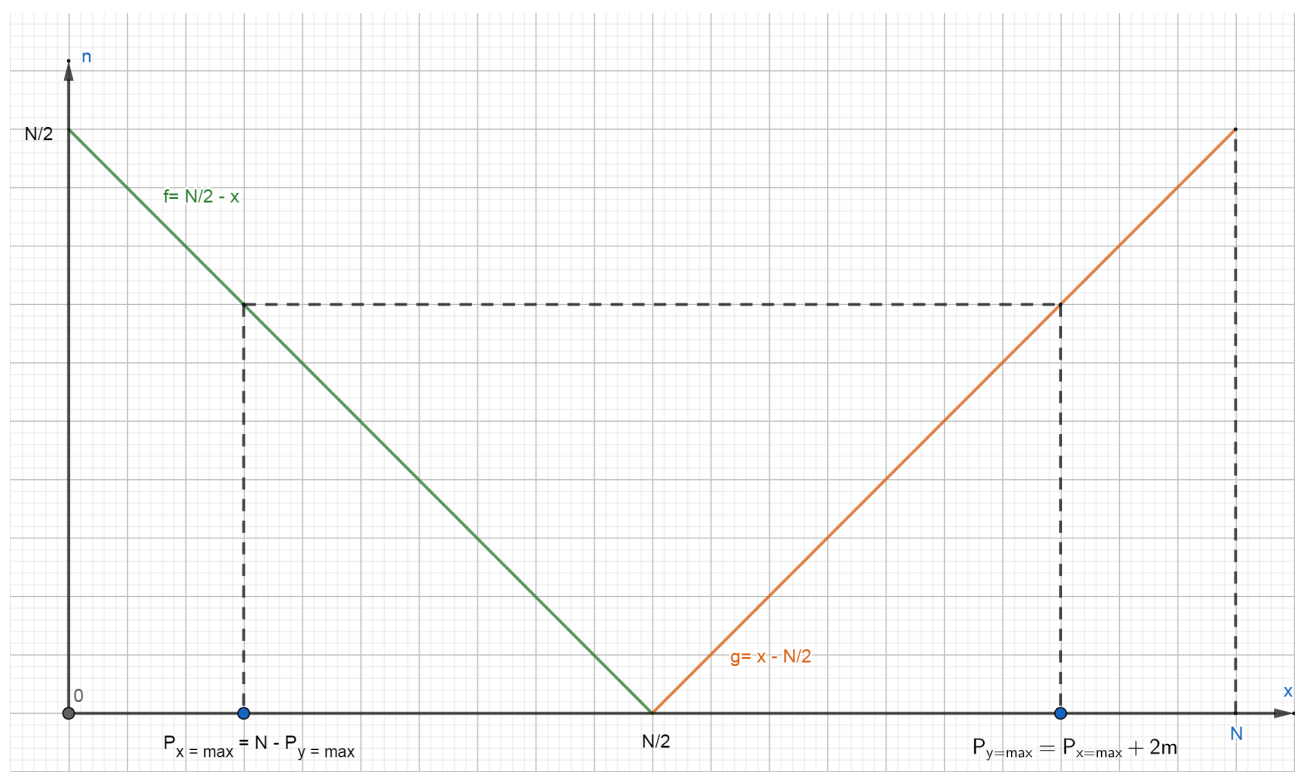

(Two graphs $f$ and $g$ are on the same coordinate system)

$\underline{\text { Important argument: }}$

We rewrite the expression (7): $N=P_{x}+\left(P_{x}+2 n\right)$.

Where $O=\left(P_{x}+2 n\right)$ with $O \in O^{*}$, and $O \in(N / 2, N-1)$. And we only focus on the value of $\left(P_{x}+2 n\right)$, because the value of $\left(P_{x}+2 n\right)$ is equivalent to the function $g=x-\frac{N}{2}$.

Applying Bertrand's theorem to the value of $\mathrm{x}$ of the function $g=x-\frac{N}{2}$, we have: There is always at least a prime number $x=P_{y}$ such that $N / 2<x=$ $P_{y}<N-1$.

- Assuming $P_{y}=P_{x}+2 m$ with $\forall P_{x}<N / 2$, and $N=P_{x=\text { const }}+P_{y=\text { const }}$. This satisfies the problem. 
- Assuming $P_{y}=P_{x}+2 m$, and $N \neq P_{x}+P_{y=\text { const }}$ with $\forall P_{x}<N / 2$. This means:

$$
\begin{gathered}
N=O^{\prime}+P_{y=\max }\left(\text { With }: O^{\prime} \in O^{*}\right) \\
==>N=P_{x=\max }+\left(2 n^{\prime}+P_{y=\max }\right)\left(\text { With }: n^{\prime} \in N^{*}\right)
\end{gathered}
$$

- In which, $P_{y=\max }$ is the largest prime number of the set $(N / 2, N-1)$; and $P_{x=\max }$ is the largest prime number of the set $\left[3, N-P_{y=\max }\right]$

To be satisfied with the expression (7), the following system of equations always has solutions with $n, n^{\prime} \in N^{*}$ :

$$
\begin{gathered}
\left\{\begin{array}{l}
N=P_{x=\max }+\left(P_{y=\max }+2 n^{\prime}\right) \\
N=P_{x=\max }+\left(P_{x=\max }+2 n\right)
\end{array}\right. \\
==>P_{y=\max }+2 n^{\prime}=P_{x=\max }+2 n\left(\text { With }: n, n^{\prime} \in N^{*}\right)
\end{gathered}
$$

Consider function $K(n)=P_{x=\max }+2 n$ with $n \in N^{*}, K(n) \in(N / 2, N-1)$ :

- We continue to apply Bertrand's theorem to the function $\mathrm{K}(\mathrm{n})$, a consequence of it has been proven as follows: "If $N>10$, there are at least 2 primes $P_{y}, P_{z} \in(N / 2, N)$ ". Therefore, in addition to the prime number $P_{y}$ defined in the function $\mathrm{K}(\mathrm{n}), \mathrm{K}(\mathrm{n})$ has at least one more prime $P_{z}$ if $P_{z} \in(N / 2, N-1)$; or $\mathrm{K}(\mathrm{n})$ only exists one prime number $P_{y}$ if $P_{z}=N-1$.

- In the case of $K(n)=P_{z} \in(N / 2, N-1)$, this leads to a contradiction with expression (11). Because if $K(n)=P_{z}=P_{y=\max }+2 n^{\prime}$ then $n^{\prime}<0 \neq N^{*}$. Therefore to satisfy the problem, if there is more than one prime $P_{y}$ in the set $(\mathrm{N} / 2, \mathrm{~N}-1)$ then $\mathrm{N}$ can always be expressed as the sum of two primes $N=P_{x}+P_{y}$

- In the case of $P_{z}=N-1$ : From the Bertrand's theorem, there always exist at least 2 primes in the set $\left(\frac{N-1}{2}, N-1\right)$. But because the set $(N / 2, N-1)$ contains only one prime $P_{y}$, so the remaining prime will be equal to $N / 2$ and $\mathrm{N}$ can be expressed as the sum of two primes $N=2 * N / 2$. (If $N / 2 \notin P^{*}$ then there will be at least two primes in the set $(N / 2, N-1)$, this case has been analyzed before). 
- Summary of analyzed cases: If $N \neq P_{x}+P_{y=\text { const }}$ with $\forall P_{x}<N / 2$ then $N=P_{x}+P_{y=\max }$; or $N=2 * P$ with $P=N / 2$.

Conclusion: For every even natural number $N>10$, it can always be expressed as the sum of two primes, with $P_{x}, P_{y} \in P^{*}$, and $P_{y}=P_{x}+2 n$.

Combining with even natural numbers $2<N \leq 10$ has been expressed as the sum of the two primes in section 3.1, leading us to prove that the Goldbach's conjecture is correct.

Proving end.

\section{References}

[1] Goldbach's conjecture - Wikipedia

[2] Bertrand's postulate - Wikipedia

[3] A Selection of Problems in the Theory of Numbers - doi.org 